Avaliação da Biodegradação em Compósitos com Fibras Naturais através de Perda de Massa e Produção de $\mathrm{CO}_{2}$

\author{
Azevedo, J. B.;* Carvalho, L. H.; Canedo, E. L.; Barbosa, J. D. V.; Silva, M. \\ W.S.
}

Rev. Virtual Quim., 2016, 8 (4), 1115-1129. Data de publicação na Web: 7 de agosto de 2016

http://rvq.sbq.org.br

\title{
Biodegradation Evaluation of Composites with Natural Fiber by Weight Loss and $\mathrm{CO}_{2}$ Production
}

Abstract: The biodegradable polymers pass by a degradation process resulting from action of microorganisms such as bacteria, fungi and algae. These polymers are being used as matrix in the composites obtained with natural fibers seeking to improve the mechanical and thermal properties without amending the biodegradability characteristics of the product. Thus, this study evaluated the biodegradation of composites obtained from commercial blends of poly (butylene adipate-coterephthalic) (PBAT) and starch as matrix and rice husk (CA) using two different techniques. PBAT starch systems with 10, 20 and $30 \%$ RH were obtained in an internal mixer and evaluated by weight loss by biodegradation test in the simulated ground, using the Sturm test. The tests for weight loss indicates that the attack of microorganisms occurs preferentially in the matrix. However, the atmosphere used in the Sturm test to evaluate the degradation was favorable biodegradation of samples of rice husk.

Keywords: Biodegradation; Composites; PBAT-starch; Rice husk.

\section{Resumo}

Os polímeros biodegradáveis passam por um processo de degradação resultante da ação de microorganismos tais como bactérias, fungos e algas. Estes polímeros estão sendo utilizados como matriz na obtenção de compósitos com fibras naturais buscando melhoria nas propriedades mecânicas e térmicas sem que altere as características de biodegradabilidade do produto. Sendo assim, este trabalho avaliou a biodegradação de compósitos obtidos com a blenda comercial de poli(butilenoadipato-co-tereftálico) (PBAT) e amido como matriz e a casca de arroz (CA) utilizando duas técnicas diferentes. Sistemas de PBAT-amido com 10, 20 e 30\% de CA foram obtidos em misturador interno e avaliados através do ensaio de biodegradação por perda de massa em solo simulado e utilizando teste de Sturm. Os ensaios por perda de massa indicam que o ataque dos micro-organismos ocorre preferencialmente na matriz. No entanto, o ambiente utilizado no teste Sturm, para avaliação da biodegradação, foi favorável a biodegradação das amostras com casca de arroz.

Palavras-chave: Biodegradação; Compósitos; PBAT-amido; Casca de Arroz.

\footnotetext{
* SENAI CIMATEC - Centro Integrado de Manufatura e Tecnologia, CEP 41650-010, Salvador-BA, Brasil.

$M$ joyce.azevedo@fieb.org.br
} DOI: 10.21577/1984-6835.20160080 


\section{Avaliação da Biodegradação em Compósitos com Fibras Naturais através de Perda de Massa e Produção de $\mathrm{CO}_{2}$} Joyce B. Azevedo, ${ }^{\mathrm{a}, *}$ Laura H. Carvalho, ${ }^{\mathrm{b}}$ Eduardo Luis Canedo, ${ }^{\mathrm{b}}$ Josiane D. V. Barbosa, ${ }^{a}$ Marivaldo Wagner S. Silva ${ }^{c}$

${ }^{a}$ Faculdade de Tecnologia SENAI CIMATEC, Campus Integrado de Manufatura e Tecnologia, Av. Orlando Gomes, 1845, Piatã, CEP 41650-010, Salvador-BA, Brasil.

${ }^{b}$ Universidade Federal de Campina Grande, Programa de Pós Graduação em Ciência e Engenharia de Materiais , Unidade Acadêmica de Engenharia de Materiais, Rua Aprígio Veloso, 882, Bairro Universitário, CEP 58429-900, Campina Grande-PB, Brasil.

${ }^{c}$ Universidade Federal da Paraíba, Centro de Ciências Aplicadas e Educação, Rua Mangueira, s/n, Centro, CEP 58297-000, Rio Tinto-PB, Brasil.

* joyce.azevedo@fieb.org.br

Recebido em 6 de agosto de 2016. Aceito para publicação em 6 de agosto de 2016

\section{Introdução}

2. Polímeros Biodegradáveis

3. Mecanismos de biodegradação e técnicas de avaliação

4. Compósitos polímeros biodegradáveis/fibras naturais

5. Experimental

5.1. Materiais

5.2. Preparação dos compósitos

5.3. Biodegradação por perda de massa em solo simulado

5.4. Avaliação da biodegradação através do teste de Sturm

\section{Resultados}

6.1. Perda de Massa

6.2. Teste de Sturm

\section{Conclusões}

\section{Introdução}

A necessidade de inovação de materiais alternativos a fim de ser adequar ao novo cenário de conscientização ambiental da sociedade e da indústria em geral tem estimulado $o$ interesse em materiais biodegradáveis. Entre estes materiais os polímeros com estas características têm sido uma alternativa para redução dos problemas 
causados pela eliminação de resíduos plásticos convencionais. Estes polímeros foram utilizados industrialmente pela primeira vez na década de 1980 na produção de filmes e outros artigos moldados e desde então constitui um mercado em constante crescimento. ${ }^{1}$ Apresentam-se com uma alternativa na busca de soluções ambientalmente favoráveis ${ }^{2}$ e tem gerado um número significativo de pesquisas, além de ser uma alternativa complementar a outros materiais utilizados, principalmente para atender à demandas específicas, por exemplo, na agricultura e indústria de embalagens. $^{3}$

Um polímero biodegradável pode encontrar uma enorme variedade de ambientes no fim da sua vida útil. Estes ambientes podem diferir em aspectos físicos (temperatura e estrutura mineral), químicos $(\mathrm{pH}$, nutrientes, oxigênio e quantidade de umidade) e biológicos (micro-organismos e enzimas). ${ }^{4,5}$ Por este motivo é relativamente difícil medir a sua biodegradabilidade, pois uma pequena variação de fatores pode gerar taxas de biodegradação completamente diferentes.

Considera-se também que características intrínsecas do polímero, como constituição química, ramificação, hifrofilicidade, estereoquímica, massa molar, cristalinidade e área superficial influem também de maneira determinante na sua taxa de biodegradação. Portanto, fatores que possam alterar as características primordiais do polímero, como a aditivação, a forma de processamento ou a mistura com outro polímero poderão afetar a sua biodegradabilidade.

Em algumas aplicações, verificam-se limitações quanto ao uso de polímeros biodegradáveis, principalmente quando existe a necessidade de melhores propriedades térmicas e mecânicas. Para promover melhorias nas propriedades desse tipo de sistemas poliméricos, tem-se buscado utilizar tecnologias como a nanotecnologia e a manufatura de compósitos com fibras lignocelulósicas. Neste contexto, e buscando a avaliação dos métodos de análise da biodegradação destes materiais, este trabalho apresenta os resultados da capacidade de biodegradação de compósitos obtidos a partir de polímero biodegradável e casca de arroz, através da avaliação de perda de massa e produção de $\mathrm{CO}_{2}$.

\section{Polímeros Biodegradáveis}

\section{Polímeros biodegradáveis são polímeros} nos quais a degradação resulta primariamente da ação de micro-organismos tais como bactérias, fungos e algas. Em geral, derivam desse processo $\mathrm{CO}_{2}, \mathrm{CH}_{4}$, componentes microbianos e outros produtos de baixa massa molar. ${ }^{6}$

O mercado mundial de polímeros biodegradáveis, quando comparado com os de polímeros convencionais, é relativamente recente. No entanto, o crescimento desse mercado é confirmado com o aumento dos números apresentados no setor. Na Europa, a produção em 2013 de polímeros biodegradáveis foi de 1,6 milhões de toneladas. Espera-se que esta produção aumente para 6,7 milhões de toneladas até 2018. ${ }^{7}$

No Brasil o mercado ainda se mostra incipiente e possui muitas dificuldades a serem superadas, como o aumento da conscientização da utilização destes polímeros em aplicações convencionais, e o alto custo quando comparado às resinas convencionais. $^{8}$

Comercialmente, já estão disponíveis misturas de polímeros biodegradáveis de diferentes origens. Estas são formuladas de forma a oferecer melhores propriedades com uma quantidade menor de materiais de alto custo. Pode-se destacar, nestas misturas, o uso de amido com poliésteres alifáticos, poli (ácido lático), poli (caprolactama) ou acetato de celulose. Nestes casos, as propriedades do amido (material de baixo custo) são melhoradas pela adição controlada de polímeros biodegradáveis. ${ }^{9}$

Dentro da classe de polímeros com características biodegradáveis podem-se 
destacar os poliésteres, eles apresentam uma estrutura que facilita o ataque por fungos que favorece a hidrólise. ${ }^{9}$

A família dos poliésteres é dividida em dois grandes grupos: poliésteres alifáticos e poliésteres aromáticos. Os poliésteres aromáticos, como o poli (tereftalato de etileno - PET), exibem excelentes propriedades mecânicas e são resistentes ao ataque de micro-organismos, esta característica se deve a presença do anel aromático que proporciona a este polímero resistência à hidrólise e a agentes químicos, dificultando o mecanismos de biodegradação. ${ }^{10}$ Por sua vez, os poliésteres alifáticos, têm suas aplicações limitadas devido às baixas propriedades mecânicas, porém são, em sua maioria, biodegradáveis. ${ }^{11}$ A biodegradabilidade apresentada nestes polímeros é devido a presença de ligações éster hidrolisáveis e, por este motivo, são facilmente atacados por fungos através de hidrólise. ${ }^{12}$

A blenda de copoliéster alifático aromático poli (tereftalato de butileno-coadipato de butineno - PBAT e amido de milho modificado com plastificante tem por nome comercial Ecobras. Este polímero tem características biodegradáveis e foi lançado comercialmente em 2007 pela BASF. Sua concepção se deve principalmente à capacidade de mistura observada no PBAT, bem como $O$ aperfeiçoamento na processabilidade e na biodegradabilidade deste polímero na forma de blenda quando misturado com amido. ${ }^{13}$

O Ecobras possui um total de $50 \%$ de matéria prima de fonte renovável na sua composição, o que ajuda a balancear o ciclo de carbono equilibrando o tempo de produção da resina com o tempo de consumo do produto obtido a partir dela. Este polímero pode ser processado em equipamentos tradicionais de transformação de injeção e extrusão podendo ainda ser aditivado. $^{14}$

O copoliéster que compõe a blenda, o PBAT, de nome comercial Ecoflex, marca registrada da empresa BASF, é um copoliéster alifático-aromático estatístico obtido a partir de butanodiol e de ácidos adípico e tereftálico via polimerização por condensação. ${ }^{9}$ A Figura 1 mostra a estrutura molecular deste polímero.

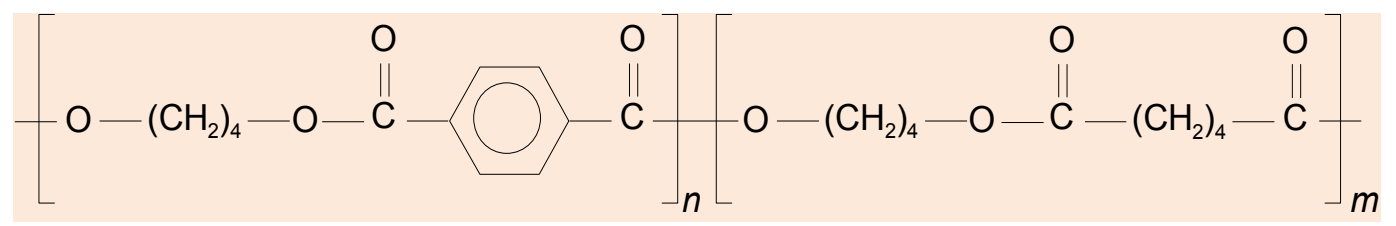

Figura 1. Estrutura molecular do PBAT

O Ecoflex foi desenvolvido especialmente para aplicações em filmes flexíveis, que são totalmente biodegradáveis, o que facilita a decomposição após o descarte de embalagens. É um polímero que apresenta características de processamento semelhante ao PEBD (polietileno de baixa densidade), sendo possível utilizar os mesmos equipamentos de fabricação de filmes de polietileno. ${ }^{15}$ Este copoliéster supera as desvantagens observadas em outros materiais alifáticos, pois combina boas propriedades mecânicas com biodegradabilidade. ${ }^{16}$

O PBAT também oferece propriedades de barreira especiais como, por exemplo, alta permeabilidade ao vapor de água, o que predestina seu uso em embalagens de frutas e vegetais, nas quais ajuda a evitar o crescimento de fungos. Suas propriedades de barreira e, sobretudo, sua compostabilidade, devem ser levadas em consideração na determinação das suas possibilidades de uso, principalmente em aplicações onde é benéfico dispor de um plástico compostável, 
resistente ao rasgamento, à prova de água, resistente à perfuração, capaz de receber impressão e permeável ao vapor de água. Ele pode ser usado na confecção de sacolas plásticas que após o uso poderão servir como sacos de lixo para rejeitos orgânicos; para filmes para agricultura e embalagens para alimentos que podem ser colocadas em recipientes para rejeitos orgânicos juntamente com restos de comida. ${ }^{17}$

Além das propriedades citadas acima, o PBAT apresenta uma boa compatibilidade com amido, celulose, lignina, PLA [Poli(ácido lático)] e PHB [Poli (hidrobutirato)], o que possibilita misturas que, por um lado, são completamente biodegradáveis e, por outro, contêm uma alta proporção de recursos renováveis. ${ }^{17}$

Dentre os materiais que possuem boa compatibilidade com o PBAT pode-se destacar o amido, polímero que também compõe a blenda Ecobras (PBAT/amido). 0 amido é um polímero natural de baixo custo que ocorre em abundância, podendo ser extraído com elevada pureza por processos industriais. ${ }^{11}$ Ele representa a maior reserva de carboidratos em plantas e pode ser digerido por humanos.

Habitualmente o amido é composto por duas macromoléculas naturais, amilose e amilopectina, cujas estruturas podem ser observadas nas Figuras 2 e 3, respectivamente. A amilose possui moléculas essencialmente lineares com massa molecular entre 105 e $106 \mathrm{~g} / \mathrm{mol}$. Em geral, há uma porcentagem em massa da ordem de $20 \%$ de amilose no amido. O segundo composto do amido, com $80 \%$ de porcentagem em massa na composição, é a amilopectina, polímero de cadeia ramificada que apresenta massa molecular entre 106 e $108 \mathrm{~g} / \mathrm{mol}$ e comprimento das ramificações entre 20 e 30 unidades de glicose. ${ }^{18,19}$

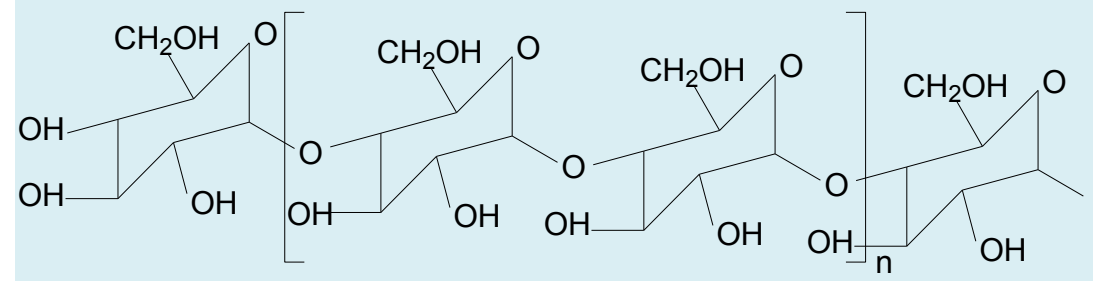

Figura 2. Estrutura molecular amilose

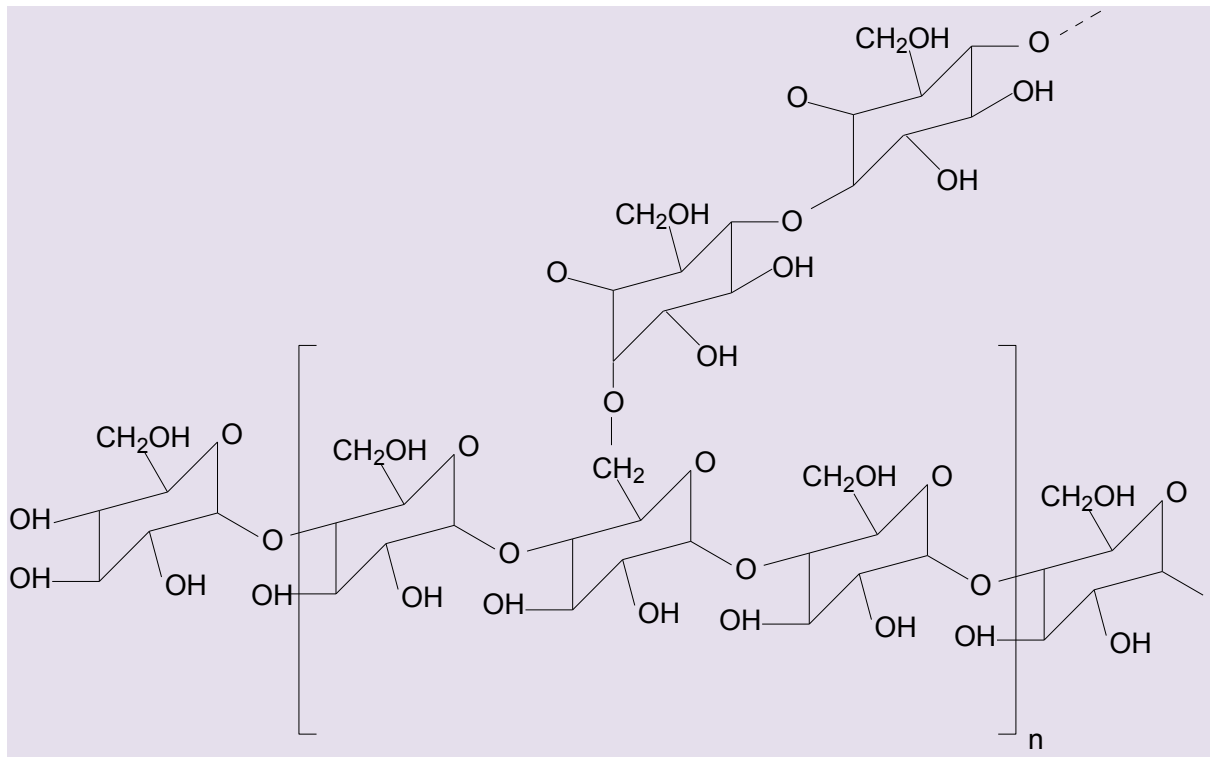

Figura 3. Estrutura molecular da amilopectina 


\section{Mecanismos de biodegradação e técnicas de avaliação}

A biodegradação em polímeros pode ocorrer tanto na presença como na ausência de oxigênio, denomidadas de biodegradação aeróbica e anaeróbica, respectivamente. Este processo acontece em duas etapas. A primeira etapa corresponde à despolimerização das macromoléculas, ocorre por meio da quebra das ligações o que resulta na fragmentação do polímero. Acontece fora do micro-organismo devido ao tamanho da cadeia polimérica e a natureza insolúvel de muitos polímeros. Reações abióticas em enzimas extracelulares são responsáveis pela quebra das cadeias poliméricas, aumentando a área de contato entre o polímero e o micro-organismo. ${ }^{20-22}$

A segunda etapa do processo de biodegradação consiste na mineralização dos fragmentos oligoméricos, onde estes são transportados para o interior dos microorganismos e transformados em biomassa. Durante o processo de mineralização são produzidos gases $\left(\mathrm{CO}_{2}, \mathrm{CH}_{4}, \mathrm{~N}_{2}\right.$ e $\left.\mathrm{H}_{2}\right)$, água, sais e novas biomassas. O processo de biodegradação ou mineralização é finalizado quando não existe mais resíduo, ou seja, o produto inicial é completamente convertido em gases e sais. ${ }^{20-22}$

Existem três elementos essenciais para que ocorra a biodegradação, são eles: ${ }^{22}$

- Micro-organismos: responsáveis pelas ações metabólicas para síntese de enzimas específicas que conseguem dar início ao processo de despolimerização e mineralizam os monômeros e oligômeros formados por este processo;

- Fatores ambientais: são essenciais para o favorecimento da ocorrência da biodegradação. Incluem temperatura, sais e umidade;

- Substrato:

corresponde principalmente à estrutura do polímero, incluindo os tipos de ligações químicas, o nível de ramificação e de polimerização, hidrofilicidade, distribuição de massa molar, cristalinidade, entre outros.

Diferentes métodos de avaliação da biodegradação de polímeros têm sido utilizados para identificar as mudanças estruturais destes materiais. Os testes são desenvolvidos com o objetivo de quantificar a capacidade dos micro-organismos degradarem esses polímeros. Dentre estes métodos de avaliação da biodegradação, a determinação de perda de massa consiste em determinar o grau e a taxa de biodegradação de materiais plásticos em exposição a solos simulados e controlados, sob condições laboratoriais, pela perda de massa da amostra submetida ao ensaio. ${ }^{12} \mathrm{O}$ monitoramento do processo de biodegradação também pode ser realizado através da medição da quantidade de $\mathrm{CO} 2$ liberada, este ensaio denominado de teste de Sturm, é considerado o mais confiável para a avaliação de biodegradabilidade de um polímero em meio microbiano ativo. ${ }^{19}$

\section{Compósitos polímeros biodegradáveis/fibras naturais}

A produção de compósitos têm se mostrado como uma das alternativas mais viáveis para o uso de polímeros biodegradáveis, já que existe a possibilidade de melhorar as propriedades físicas e mecânicas de produtos obtidos com estes polímeros a partir da incorporação de um reforço em uma matriz homogênea. ${ }^{23}$

Estes materiais surgiram como uma forma de substituição dos compósitos poliméricos tradicionais e buscam a redução do impacto ambiental causado pelo descarte rápido de produtos que são usualmente produzidos com estes materiais. Podem ser produzidos a partir de fibras naturais com polímeros com ou sem características biodegradáveis. ${ }^{24}$ No entanto, os compósitos que combinam fibras naturais e polímeros biodegradáveis apresentam melhores características do 
ponto de vista ambiental por serem degradáveis e sustentáveis.

Os compósitos utilizando polímeros biodegradáveis e fibras naturais, chamados na literatura muitas vezes de "compósitos verdes", têm sido utilizados com eficácia em muitas aplicações, sejam elas em produtos de rápido descarte, assim como em produtos com vida útil maior. ${ }^{25}$ Apresentam completa degradação no solo ou na compostagem e têm recebido uma grande atenção, visando o desenvolvimento de materiais que conservem a propriedade de biodegradação e apresentem melhores propriedades mecânicas. $^{26}$

Com base nas informações acima, o presente trabalho avaliou a biodegradação de compósitos obtidos a partir da blenda PBAT/amido com casca de arroz utilizando dois métodos de avaliação, perda de massa e produção de $\mathrm{CO}_{2}$.

\section{Experimental}

\subsection{Materiais}

Para produção dos compósitos foi utilizado como matriz o Ecobras RD 704, uma blenda com $48 \%$ de PBAT e $52 \%$ de amido, fornecida pela BASF. Como fase dispersa, foi utilizada, em diferentes concentrações, casca de arroz micronizada fornecida pela Cerealista Polisul com partículas variando de tamanho entre 275 e 512,5 $\mu \mathrm{m}$.

\subsection{Preparação dos compósitos}

Os compósitos com matriz de PBAT-amido foram processados em misturador interno Haake Rheomix 3000 com 10, 20 e 30 \% em massa de casca de arroz. Devido as suas características higroscópicas, os insumos foram secos em estufa antes do processamento. Utilizou-se temperatura de $100{ }^{\circ} \mathrm{C}$ para secar a casca de arroz durante 4 horas. Os parâmetros de secagem do PBATamido foram sugeridos pelo fornecedor; utilizou-se $80{ }^{\circ} \mathrm{C}$ por 2 horas. Após secagem, os materiais foram pré-misturados por tamboreamento antes de serem alimentados nos equipamentos de processamento.

No misturador utilizou-se rotores de alta intensidade (tipo Roller) a 60 rotações por minuto (rpm) por 10 minutos. Após mistura, o material foi triturado em um moinho de facas e seco a $60{ }^{\circ} \mathrm{C}$ por 4 horas para preparação dos corpos de prova.

Os corpos de provas utilizados nesta análise, com dimensões de 2,0 cm x 2,0 cm x $0,3 \mathrm{~cm}$, foram obtidos em prensa hidráulica do tipo uniaxial. Durante a prensagem utilizou-se temperatura de $170^{\circ} \mathrm{C}$, inicialmente realizou-se uma pré-prensagem durante 2 minutos, após está etapa aumentou-se a pressão para 3 toneladas e após 5 minutos para 6 toneladas permanecendo nesta condição por mais 5 minutos.

O fluxograma da Figura 4 ilustra as etapas de obtenção dos compósitos. 


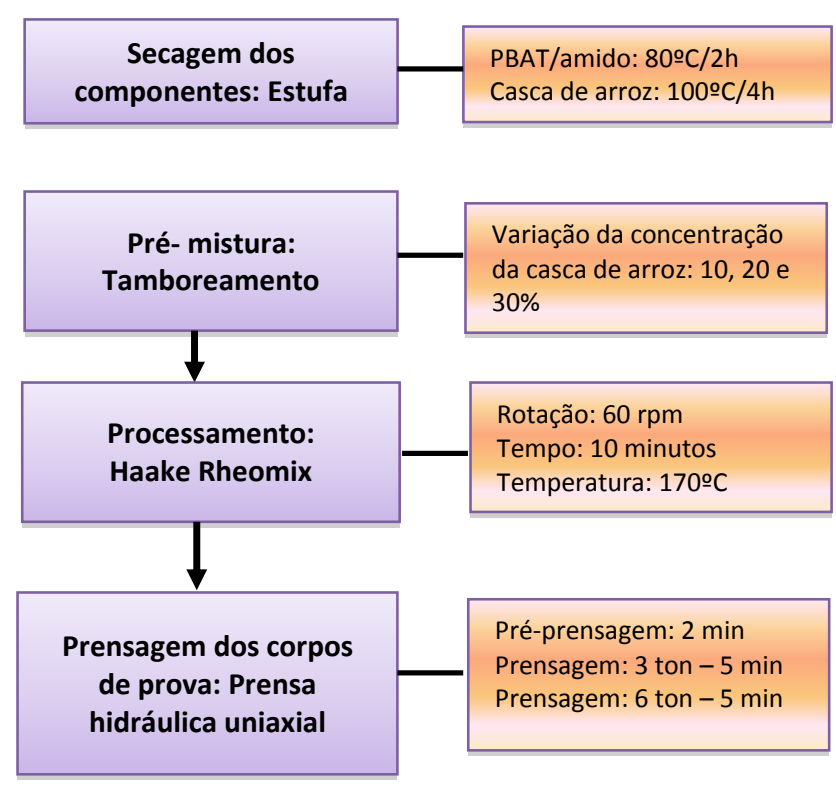

Figura 4. Etapas para obtenção dos compósitos

\subsection{Biodegradação por perda de massa em solo simulado}

O ensaio de biodegradação por perda de massa foi baseado na norma ASTM G 160-03. O solo utilizado possuiu $2 \mathrm{~kg}$ de esterco de cavalo seco, $2 \mathrm{~kg}$ de areia grossa e $2 \mathrm{~kg}$ de solo fértil com baixo teor de argila. Após mistura destes constituintes, a terra foi armazenada em um recipiente coberto por três meses para maturação.

Passado o período de maturação, dividiuse a mistura em 12 béqueres onde as amostras foram enterradas. Para cada formulação foram enterradas três amostras.

A Figura 5 ilustra o procedimento utilizado para enterrar os corpos de prova no béquer.

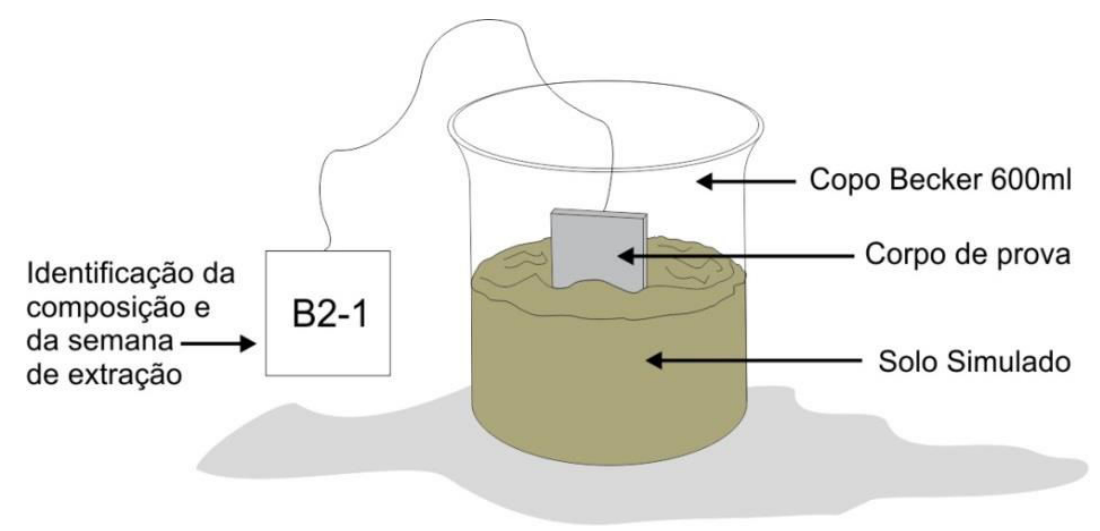

Figura 5. Corpos de provas enterrados em Becker para análise de biodegradação

Os béqueres com as amostras foram colocados em estufa projetada por Silva (2012). ${ }^{27}$ O projeto desta estufa contempla todos os aspectos, parâmetros e valores da norma de biodegradação ASTM G-160-03. ${ }^{28}$

A Figura 6 mostra a disposição dos 
béqueres com as amostras na estufa 150 e 180 dias.

utilizada.

A morfologia das amostras degradadas foi

A perda de massa foi avaliada nas amostras através da pesagem inicial, em tempo zero do ensaio de biodegradação, e pesagem em $7,14,21,28,30,60,90,120$, avaliada por cinco meses através de microscopia eletrônica de varredura (Shimadzu, mod. SSX-550).

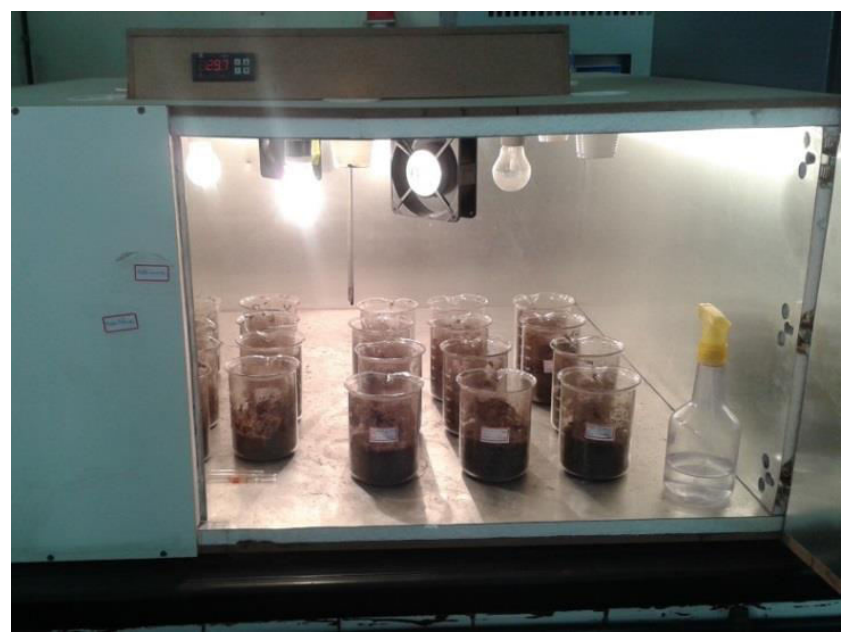

Figura 6. Estufa de biodegradação

\subsection{Avaliação da biodegradação através de teste de Sturm}

A avaliação da biodegradação utilizando o teste de Sturm foi realizada utilizando o mesmo solo do teste de biodegradação por perda de massa. O teste foi realizado baseado na norma ASTM D 5338-98. Utilizouse um sistema composto por um compressor de ar, um reator e dois recipientes coletores de $\mathrm{CO}_{2}$, um colocado antes e outro após o reator, todos conectados por mangueira, contendo 400 e $200 \mathrm{~mL}$, respectivamente, de solução de hidróxido de bário. A Figura 7 ilustra o sistema utilizado durante o teste.

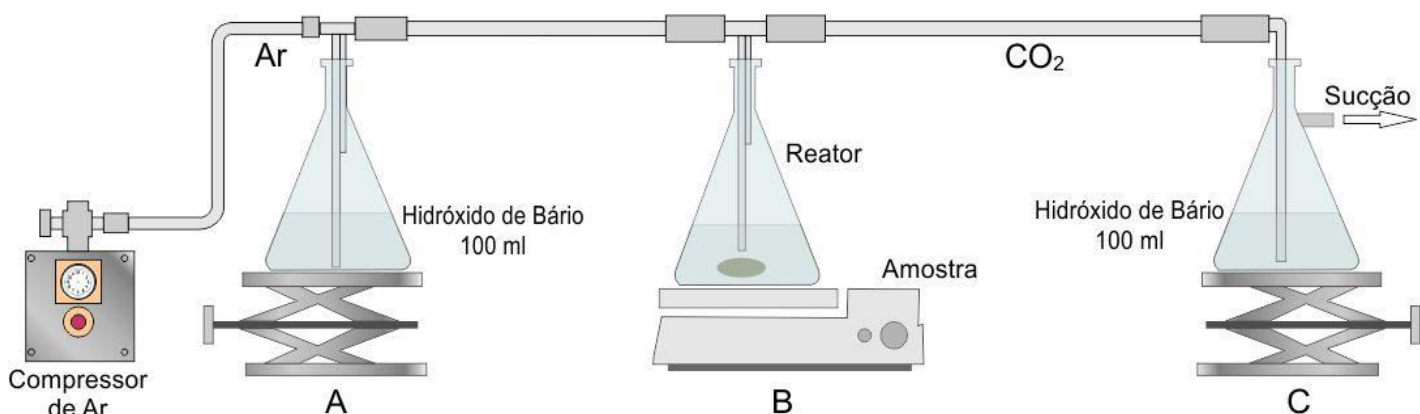

Figura 7. Esquema de montagem para realização do Teste de Sturm (adaptação da ref. 23)

As soluções de $\mathrm{Ba}(\mathrm{OH})_{2}$ foram preparadas dissolvendo-se 4,8 e 3,2 g do reagente em 400 e $200 \mathrm{~mL}$ de água destilada, respectivamente, para o primeiro e último recipientes.

No reator foram introduzidos $200 \mathrm{~mL}$ do solo utilizado no ensaio de perda de massa. 
Neste solo foi realizada a ativação dos microorganismos. Para tanto, os erlenmeyers com o solo foram submetidos a aquecimento $\left(100^{\circ} \mathrm{C}\right)$ por 10 minutos. Após esse período o sistema foi resfriado a temperatura ambiente para início do ensaio.

Durante o teste, a cada 24 horas, o último recipiente do sistema foi substituído por outro contendo uma nova solução de hidróxido de bário, e o conteúdo do recipiente substituído, filtrado a vácuo. 0 filtrado foi titulado com ácido clorídrico (1 $\mathrm{mol} / \mathrm{L}$ ) para determinação da produção de $\mathrm{CO}_{2}$ por diferença, isto é, por determinação do $\mathrm{Ba}(\mathrm{OH})_{2}$ em excesso e consequente obtenção do $\mathrm{Ba}(\mathrm{OH})_{2}$ precipitado como $\mathrm{BaCO}_{3}$, que é diretamente proporcional ao $\mathrm{CO}_{2}$ produzido, de acordo com as equações 1 e 2:

$$
\begin{array}{ll}
\mathrm{Ba}(\mathrm{OH})_{2(\mathrm{aq})}+\mathrm{CO}_{2(\mathrm{~g})} \rightarrow \mathrm{BaCO}_{3(\mathrm{~s})}+\mathrm{H}_{2} \mathrm{O}_{(\mathrm{l})} & \text { Eq. } 1 \\
\mathrm{Ba}(\mathrm{OH})_{2(\mathrm{~s})(\text { exc. })}+2 \mathrm{HCl}_{(\text {(aq) }} \rightarrow \mathrm{BaCl}_{2(\mathrm{~s})}+2 \mathrm{H}_{2} \mathrm{O}_{(!)} & \text {Eq. } 2
\end{array}
$$

Realizou-se, neste ensaio, o branco, cujo procedimento foi idêntico ao descrito anteriormente, sem a presença dos compósitos.

A população microbiana do solo também foi investigada. Sendo assim, inicialmente obteve-se uma suspensão dos microorganismos através da diluição de 1 grama do solo diluído em água estéril. Esta suspensão foi transferida para um tubo de ensaio contendo um caldo glicosado e então submetida à água fervente por 5 minutos. Após resfriamento, $1 \mathrm{~mL}$ do caldo foi transferido para um tubo com $9 \mathrm{~mL}$ de água estéril e então submetido a agitação para homogeneização. Este material foi transferido para placas estéreis que continham $15 \mathrm{~mL}$ de Agar Nutritivo fundido e resfriado a $45^{\circ} \mathrm{C}$. $\mathrm{O}$ meio então foi agitado para homogeneização e após solidificação as placas foram invertidas e incubadas a temperatura ambiente por 48 horas.

Após 48 horas, colônias selecionadas foram transferidas com alça de platina para um tubo com Agar Nutritivo, e incubadas à temperatura ambiente.

\section{Resultados}

\subsection{Perda de Massa}

Os resultados de perda de massa para as amostras submetidas à biodegradação em solo simulado estão apresentados na Figura 8. Ao contrário do esperado, foi observado um aumento de massa após 7 dias de enterro das amostras. Acredita-se que esse aumento de massa esteja relacionado a uma absorção inicial de água por parte das amostras. Em tempos mais longos, como esperado, verificou-se uma redução progressiva de massa das amostras, o que foi atribuído à biodegradação. Segundo estudos realizados por Witt et al. $(2001)^{16}$, a biodegradação do PBAT praticamente se completa (99,9 \%) decorridos 22 dias de enterro em solo. A biodegradação sofrida pelo PBAT-amido, no estudo aqui apresentado, foi bem mais lenta. No caso aqui exposto, após 30 dias de enterro em solo simulado, a perda de massa observada foi de aproximadamente $10 \%$ e, após 180 dias, foi entre 25 e $30 \%$. 


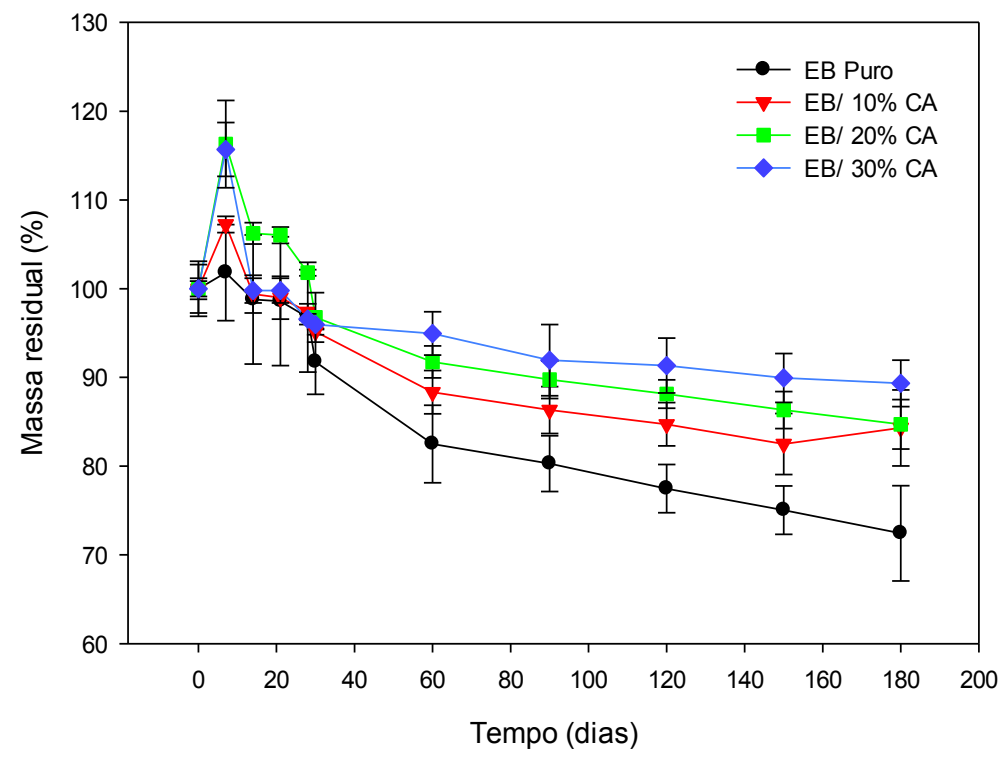

Figura 8. Perda de massa para em função do tempo de biodegradação em solo

Os resultados indicam que a perda de massa foi maior para o polímero puro, ou seja, os compósitos com casca de arroz mostraram-se mais resistentes à biodegradação do que a matriz polimérica pura. Completados 6 meses de enterro em solo, foram observadas reduções de massa médias de 27, 22, 17 e 15\% para o PBATamido (Ecobras) puro e para os compósitos com 10, 20 e $30 \%$ de casca de arroz, respectivamente.

A Figura 9 mostra as microscopias das amostras de polímero puro e dos compósitos submetidos a biodegradação.

Verifica-se através das microscopias das amostras degradadas, que a biodegradação no intervalo de tempo estudado ocorre principalmente no polímero. Portanto, com a adição da casca de arroz, ocorreu uma perda de massa percentual menor nos compósitos, mesmo que tenha havido um aumento na biodegradação.

\subsection{Teste de Sturm}

A Figura 10 apresenta os resultados da produção de $\mathrm{CO}_{2}$. Os resultados indicam que uma quantidade maior de $\mathrm{CO}_{2}$ é liberada com a incorporação da casca de arroz e que o teor de $\mathrm{CO}_{2}$ liberado parece aumentar ligeiramente com o teor de casca de arroz adicionada ao sistema. Para melhor apreciar os resultados obtidos com este ensaio, foi calculada a taxa de biodegradação das amostras ensaiadas durante 35 dias (Tabela $1)$. 


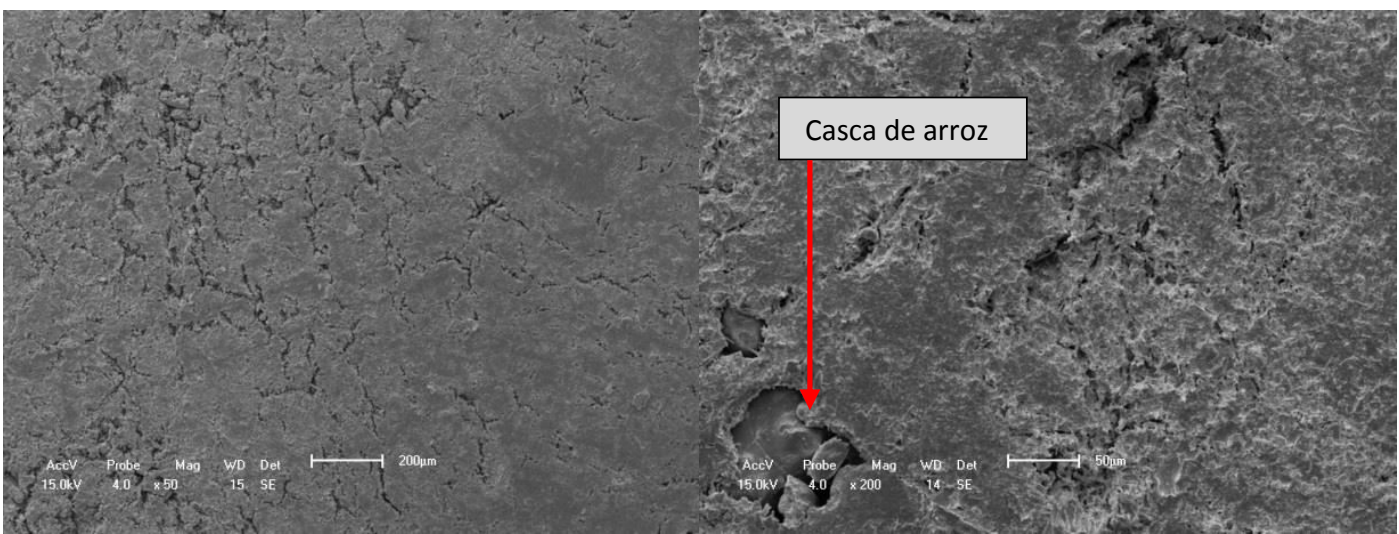

(a)

(b)

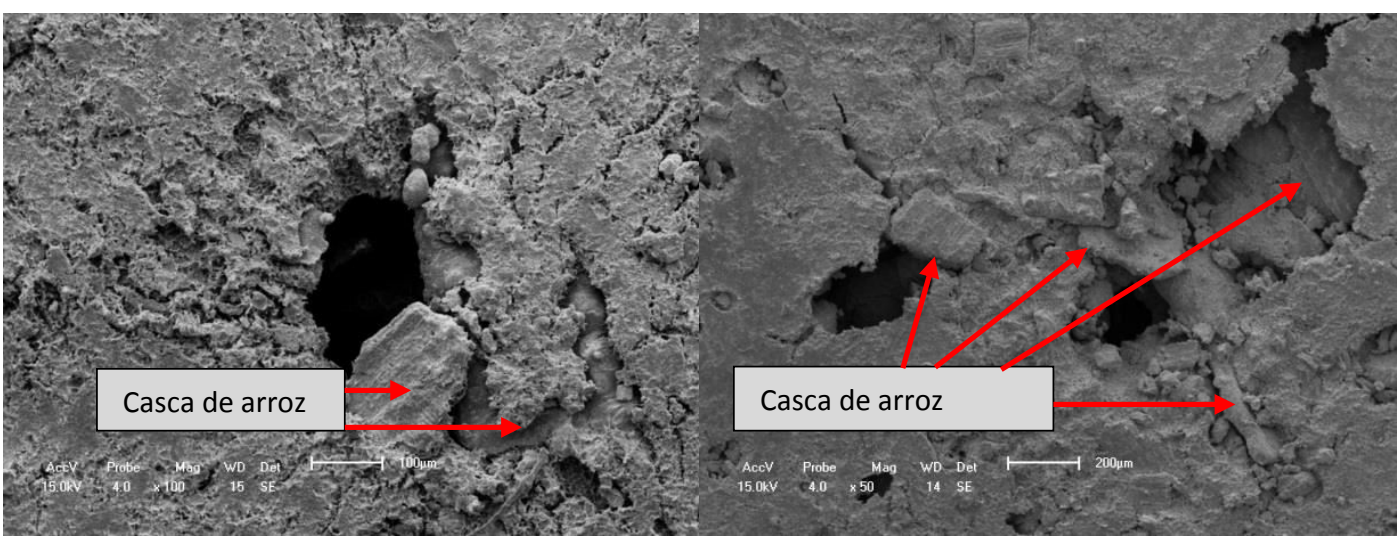

(c)

(d)

Figura 9. Morfologia de amostras do polímero puro e dos compósitos submetidos a biodegradação por 5 meses: a) EB Puro; b)EB/10\% CA; c)EB/20\%CA; d)EB/30\%CA

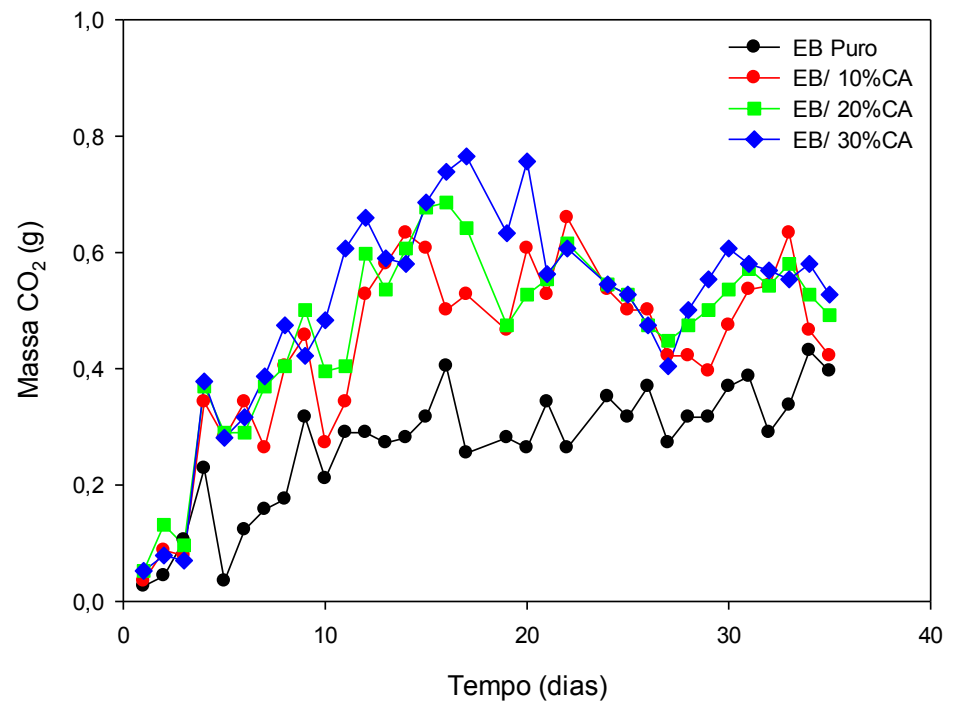

Figura 10. Produção de $\mathrm{CO}_{2}$ em função do tempo de biodegradação para compósitos PBATamido/casca de arroz 
Tabela 1. Taxa de biodegradação de compósitos PBAT-amido/casca de arroz

\begin{tabular}{|c|c|}
\hline Amostras & $\begin{array}{c}\text { Taxa de biodegradação } \\
\text { (g de } \mathrm{CO}_{2} / \text { dia) }\end{array}$ \\
\hline EB puro & 0,25 \\
\hline $\mathrm{EB} / 10 \% \mathrm{CA}$ & 0,41 \\
\hline $\mathrm{EB} / 20 \% \mathrm{CA}$ & 0,44 \\
\hline $\mathrm{EB} / 30 \% \mathrm{CA}$ & 0,46 \\
\hline
\end{tabular}

Apesar de não terem sido observadas variações significativas para taxa de biodegradação dos compósitos, esta tendeu a aumentar com o aumento da concentração de casca de arroz. A taxa de biodegradação do compósito com casca de arroz é praticamente duas vezes maior do que a do polímero puro.

Estudos realizados por Rosa \& Filho $(2003)^{19}$ mostram que a taxa de biodegradação do amido, avaliada através do teste de Sturm, torna-se maior após 110 dias. Segundo os autores, a adição de amido em PHB, PHBV (Poli (hidroxibutirato-cohidrovalerato)) e PCL (Poli (caprolactana)) provocou aumento na taxa de biodegradação de todas as blendas. Esse aumento pode ser atribuído ao fato das ligações $\left(\alpha-1,6^{\prime}\right)$ glicosídicas presentes no amido serem mais facilmente hidrolisáveis e consequentemente biodegradáveis.
No ensaio realizado neste trabalho, a massa de $\mathrm{CO}_{2}$ liberada pelos sistemas aumentou com a incorporação da casca de arroz, o que indica que a incorporação da casca de arroz acelera a biodegradação do sistema e que ambos, PBAT-amido e casca de arroz, se degradam sob as condições experimentais adotadas no teste de Sturm.

Os resultados obtidos com este teste indicam que o ambiente ao qual a amostra foi submetida no teste de Sturm favorece também a decomposição dos constituintes (celulose, hemicelulose e lignina) da casca de arroz, acelerando assim a liberação de $\mathrm{CO}_{2}$. Este processo de biodegradação foi favorecido pela diversidade da flora microbiana presente no solo utilizado no teste de Sturm (Figura 11). Verificaram-se colônias de fungos, bactérias e leveduras. A presença desses microrganismos mostra que o solo está viável para o estudo de biodegradação.

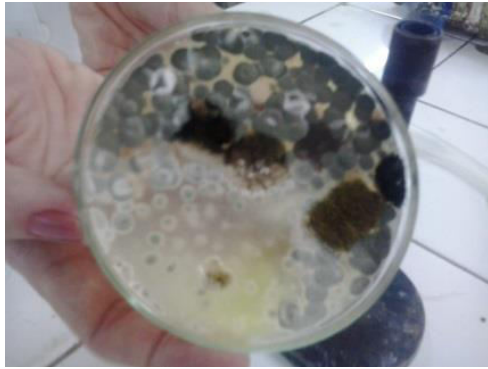

(a)

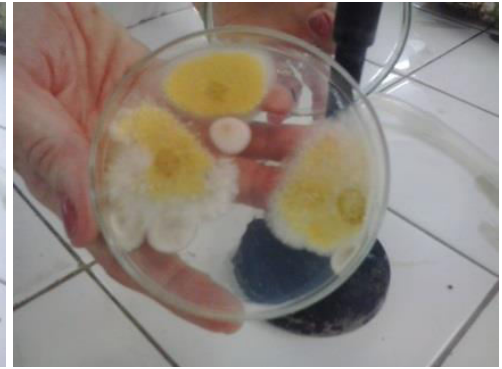

(b)

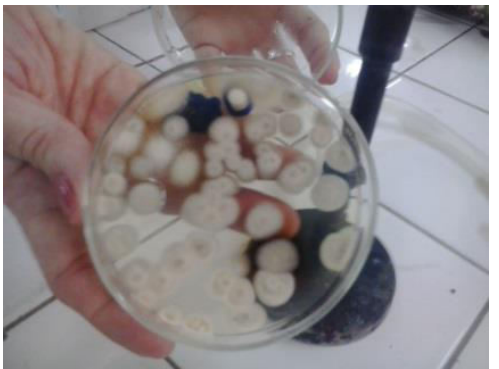

(c)

Figura 11. Flora microbiana presente no solo: a) Colônias de fungos; b) Colônias de bactérias; c) Colônias de leveduras

A investigação da população microbiana indicou que o solo utilizado no ensaio de perda de massa, por outro lado, continha uma população microbiana pouco ativa. 
Possivelmente este comportamento deve-se a forma de armazenamento e condicionamento ao qual o solo utilizado no teste de perda de massa foi submetido. Após realização do choque térmico no solo, para realização do teste de Sturm, os esporos passaram para a forma vegetativa e passaram a crescer e atuar na biodegradação.

O ensaio de perda de massa pode ter sido muito influenciado pela absorção de umidade (Figura 8). Para os compósitos, a umidade absorvida pelo sistema resultou em uma perda de massa menor, influenciando no resultado nesta técnica.

\section{Conclusões}

O uso de casca de arroz como elemento de reforço é uma forma de reaproveitamento de um resíduo agrícola e os compósitos produzidos não só são ecologicamente viáveis, mas também podem contribuir para a pesquisa acadêmica, já que são poucos os trabalhos realizados com estes materiais na forma de compósitos que utilizam matriz polimérica com características biodegradáveis.

A avaliação do efeito de biodegradação da casca de arroz em compósitos com PBATamido pode ser realizada por duas técnicas diferentes, sendo que os ensaios de biodegradação por perda de massa mostram que a biodegradação nos compósitos PBATamido/casca de arroz ocorre preferencialmente no polímero. Neste sistema a presença da casca de arroz resulta em menor perda de massa. A avaliação da biodegradação através de teste de Sturm indicou uma maior produção de $\mathrm{CO}_{2}$ para as amostras com casca de arroz, o que mostra o favorecimento da degradação das amostras no ambiente ao qual o ensaio foi realizado.

\section{Agradecimentos}

Os autores agradecem a BASF, a Corn Products, a Cerealista Polisul e ao Departamento de Química da UFPE.

\section{Referências Bibliográficas}

${ }^{1}$ Mokhothu, T. H.; John, M. J. Review on hygroscopic aging of cellulose fibres and their biocomposites. Carbohydrate Polymers 2015, 131, 337. [CrossRef]

${ }^{2}$ Rhim, J. W.; Park, H. M.; Ha, C. S. Bionanocomposites for food packaging applications. Progress in Polymer Science 2013, 38, 1629. [CrossRef]

${ }^{3}$ Manal, D.; Zeid, A.; Muller, R. J.; Deckwer, W. D. Biodegradation of Aliphatic Homopolyesters and Aliphatic-Aromatic Copolyesters by Anaerobic Microorganisms. Biomacromolecules 2004, 5, 1687. [CrossRef] [PubMed]

${ }^{4}$ Hrenović, J.; Kovač, V.; Ivanković, T. Microbial colonization of high density polyethylene garbage bags. Hrvatski Časopis Za Prehrambenu Tehnologiju, Biotehnologiju I Nutricionizam 2011, 6, 52.

${ }^{5}$ van der Zee, M. Analytical methods for monitoring biodegradation processes of environmentally polymers. Handbook of Biodegradable Polymers: Isolation, Synthesis, Characterization and Applications 2011, 263. [Link]

${ }^{6}$ Azevedo, J. B.; Tese de Doutorado, Universidade Federal de Campina Grande, 2013.

${ }^{7}$ European Bioplastics. Disponível em: http://en.european-bioplastics.org/. Acesso em: 14 Maio 2015.

${ }^{8}$ Lancellotti, A.; Bioplastics in Brazil: Beyond the Green Speech, Frost \& Sullivan, 2010. [Link]

${ }^{9}$ Smith, R.; Biodegradable polymers for industrial applications, New York: CRC Press, 2005. [CrossRef] 
${ }^{10}$ Barbosa, J. D. V; Tese de Doutorado, Universidade Federal de Campina Grande, 2012.

${ }^{11}$ Pellicano,. M.; Dissertação de Mestrado, Universidade Federal de São Carlos, 2008. [Link]

${ }^{12}$ Rosa, D. S., Filho, R. P.; Biodegradação: um ensaio com polímeros. Moara Editora: Bragança Paulista, 2003.

${ }^{13}$ Vieira, M. M. G.; Dissertação de Mestrado, Universidade Federal de São Carlos, 2010. [Link]

${ }^{14}$ Harada, J.; Biopolímeros Biodegradáveis e Compostáveis Em: Palestra Técnica- SENAI CIMATEC, Salvador, 2008.

${ }^{15}$ Yamamoto., M; Witt, U.; Skupin. G.; Dieter, B. D.; Muller., R.-J.; Biodegradable Aliphaticaromatic Polyesters: ECOFLEX ${ }^{\circledR}$. Biopolymers Online, 2005. [CrossRef]

16 Jacquel, N.; Saint-Loup, R.; Pascault, J. P.; Rousseau, A.; Fenouillot, F. Bio-based alternatives in the synthesis of aliphaticaromatic polyesters dedicated to biodegradable film applications. Polymer 2015, 59, 234. [CrossRef]

${ }^{17}$ Reimer, V.; Kunkel, A.; Philipp, S. Avaliação da ecoficiência de polímeros obtidos a partir de fontes renováveis. Plástico Industrial 2010. [Link]

18 Halley, P. Thermoplastic starch biodegradable polymers. In: Biodegradable polymers for industrial applications, Boca Raton: SMITH, R., 140-159, 2005. [CrossRef]

${ }^{19}$ Rosa, D. S.; Filho, R. P.; Chui, Q. S. H.; Calil, M. R.; Guedes, C. G. F. The biodegradation of poly-b-(hydroxybutyrate), poly-b(hydroxybutyrate-co-b-valerate) and poly(ecaprolactone) in compost derived from municipal solid waste. European Polymer Journal 2003, 39, 233. [CrossRef]

${ }^{20}$ Fechine, G. J. M. A era dos polímeros biodegradáveis. Revista Plástico Moderno 2010, 423. [Link]

${ }^{21}$ Luckachan, G. E.; Pillai, C. K. S. Biodegradable polymers-a review on recent trends and emerging perspectives. Journal of Polymers and the Environment 2011, 19, 637. [CrossRef]

${ }^{22}$ Denchev, Z. Z.; Biodegradation studies of polymer blends and composites comprising biopolymers Em: Handbook of Engineering Biopolymers: Homopolymers, Blends and Composites, Hanser, 2007. [CrossRef]

${ }^{23}$ Satyanarayana, K. G., Arizaga, G. C., Wypych, F. Biodegradable composites based on lignocellulosic fiber-Na overview. Progress in Polymer Science 2009, 34, 982. [CrossRef]

${ }^{24}$ Mohanty, A. K.; Misra, M.; Drzal, L. T.; Natural Fibers, Biopolymers, and Biocomposites, Taylor \& Francis: Boca Raton, 2005. [CrossRef]

${ }^{25}$ John, M. J.; Thomas, S. Review: Biofibres and biocomposites. Carbohydrate Polymers 2008, 71, 343. [CrossRef]

${ }^{26} \mathrm{Wu}, \quad$ C. Process, Characterization and Biodegradability of Aliphatic Aromatic Polyester/Sisal Fiber Composites. Journal of Polymers and the Environment 2011, 19, 706. [CrossRef]

${ }^{27}$ Silva, M. W. S.; Tese de Doutorado, Universidade Federal de Campina Grande, 2012.

${ }^{28}$ Vinhas, G. M.; Almeida, Y. M. B.; Lima, M. A. G. A. Estudo das propriedades e biodegradabilidade de blendas de poliésteres/amido submetidas ao ataque microbiano. Química Nova 2007, 30, 1584. [CrossRef] 